\title{
Digital Predistortion of Ultra-Broadband mmWave Power Amplifiers with Limited Tx/Feedback Loop/Baseband Bandwidth
}

\author{
Chao Yu (D), Qianyun Lu, Honglei Sun, Xingwang Wu, and Xiao-Wei Zhu \\ State Key Laboratory of Millimeter Waves, School of Information Science and Engineering, Southeast University, Nanjing 210096, China \\ Correspondence should be addressed to Chao Yu; chao.yu@seu.edu.cn
}

Received 2 November 2017; Revised 9 January 2018; Accepted 13 February 2018; Published 19 March 2018

Academic Editor: Christian Fager

Copyright (c) 2018 Chao Yu et al. This is an open access article distributed under the Creative Commons Attribution License, which permits unrestricted use, distribution, and reproduction in any medium, provided the original work is properly cited.

\begin{abstract}
A novel digital predistortion (DPD) technique is proposed to linearize ultra-broadband millimeter wave (mmWave) power amplifiers (PAs) by only employing very limited bandwidth resources for the Tx, feedback loop (FB), and baseband (BB). Compared to the conventional methods, the proposed method will comprehensively reduce the bandwidth requirements for the whole system, which will make the linearization affordable for mmWave PAs. To validate the proposed idea, a 4-carrier $320 \mathrm{MHz}$ modulated signal was employed to excite a mmWave PA with the center frequency of $41 \mathrm{GHz}$. Experimental results have proven that the proposed method can effectively realize the PA linearization with very narrow $\mathrm{Tx} / \mathrm{FB} / \mathrm{BB}$ bandwidth, which largely extends the capability of $\mathrm{DPD}$ to the forthcoming $5 \mathrm{G}$ era.
\end{abstract}

\section{Introduction}

The fifth-generation (5G) communication systems have gradually attracted more and more attentions from both academia and industry. Since wideband spectrum resource is required to support the ultimate goal of $10 \mathrm{Gbps}$ [1], millimeter wave (mmWave) frequency band is one of the most promising candidates in $5 \mathrm{G}$ frequency plan, as shown in Figure 1(a). Many countries have gradually released their drafts for the mmWave frequency band allocations around $28 \mathrm{GHz}$ and $40 \mathrm{GHz}$ [2]. To fully exploit the advantage of mmWave technique, ultrabroadband modulated signal, for example, $500 \mathrm{MHz}$, will be employed to enable high-speed transmission. However, this change will place a huge burden on RF circuit design, especially for the linearization of ultra-broadband mmWave power amplifiers (PAs).

Usually, the PAs need to operate at nonlinear region to achieve high efficiency, which will incur not only the inband distortion, but also the out-of-band (OOB) spectrum regrowth [3]. To overcome this problem, the digital predistorter (DPD) has widely become a crucial block in modern wireless transmitters [4], as shown in Figure 1(b). This module can effectively remove the nonlinear distortion with very high accuracy but low cost. With the development of
DPD technique, many effective models have been proposed in recent years [5], such as dynamic deviation reductionbased Volterra series model (DDR) [6], decomposed vector rotation-based model (DVR) [7, 8], memory polynomial model (MP) [9], and generalized memory polynomial model (GMP) [10]. Usually, in baseband (BB) processing, the DPD characteristics will be modeled by the nonlinear operators, which normally occupy multiple times the input bandwidth. For example, the 5th-order nonlinear operator will occupy $5 \mathrm{x}$ the input bandwidth. Thus, the bandwidth for the transmitter chain (Tx) should be wide enough to support such $5 \mathrm{x}$ the input bandwidth. Furthermore, in order to realize accurate model extraction, the bandwidth of the PA output will also keep $5 \mathrm{x}$ the input bandwidth to capture all major nonlinear distortion in the feedback loop (FB) [11]. Therefore, in narrow-band scenarios, these bandwidth requirements for $\mathrm{Tx} / \mathrm{FB} / \mathrm{BB}$ are affordable and thus the DPD systems work well and have been widely deployed.

However, with the mmWave era coming, the modulated bandwidth has been quickly increased in $5 \mathrm{G}$ wireless systems as shown in Figure 1(a). Suddenly, the DPD systems are facing huge bandwidth limitation as shown in Figure 1(b). Not only will the system require the Tx and feedback loop 


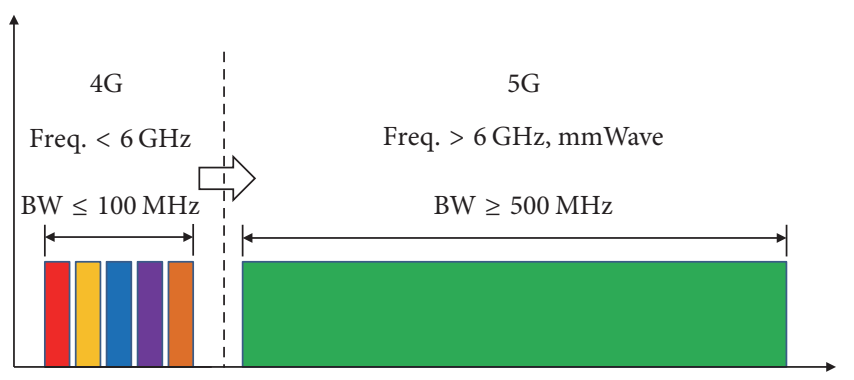

(a)

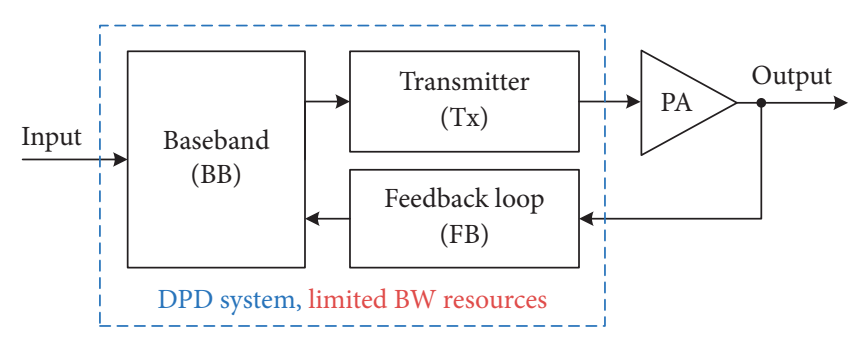

(b)

Figure 1: (a) The frequency and bandwidth of 5G scenario. (b) The challenge on DPD system for ultra-broadband mmWave wireless communication system.

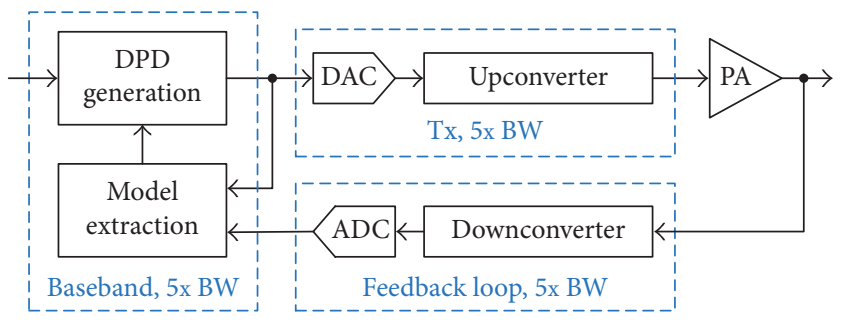

(a)

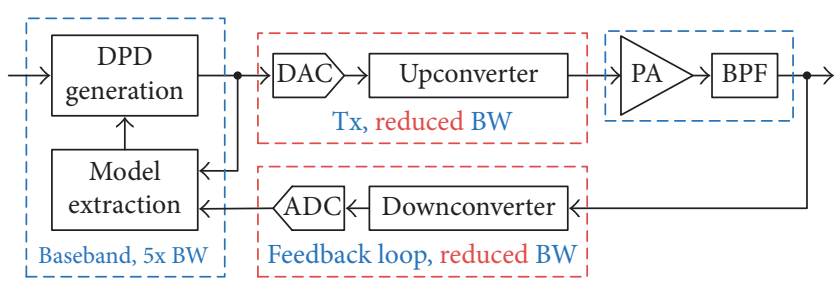

(c)

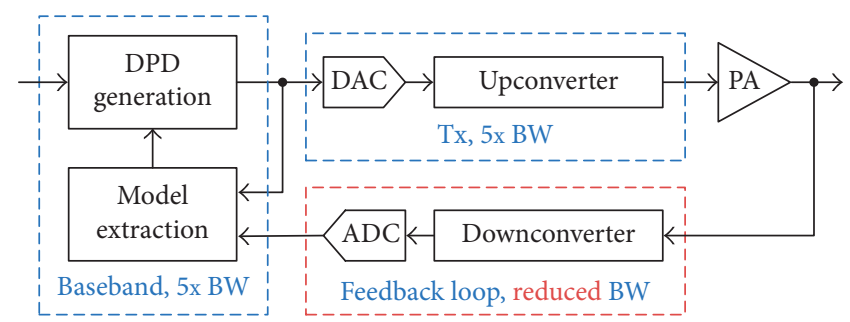

(b)

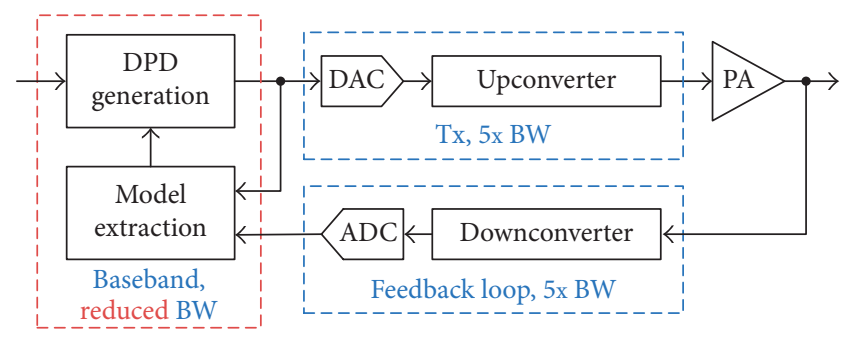

(d)

Figure 2: Available resources for DPD systems. (a) Conventional DPD system. (b) DPD system with reduced FB bandwidth. (c) DPD system with reduced Tx/FB bandwidth. (d) DPD system with reduced BB bandwidth.

with wider bandwidth, but also the baseband processing units, such as FPGA, will also need high bandwidth to deal with high-speed data. Apparently, it is not cost-effective for mmWave scenarios to directly employ the conventional methods.

In this paper, a comprehensive solution for these three bandwidth limitations will be proposed to further extend the DPD's capability into the linearization of mmWave PAs. The proposed technique only employs the limited $\mathrm{Tx} / \mathrm{FB} / \mathrm{BB}$ bandwidth, that is, $1 \mathrm{x} \sim 2 \mathrm{x}$ the original input bandwidth, but can achieve similar linearization performance. Furthermore, this technique will be validated by an mmWave PA centered at $41 \mathrm{GHz}$ and excited with a modulated signal with $320 \mathrm{MHz}$ bandwidth.

The rest of the paper is organized as follows. In Section 2, the DPD system with reduced $\mathrm{Tx} / \mathrm{FB} / \mathrm{BB}$ bandwidth will be discussed. Then, a novel solution will be proposed and explained in detail in Section 3. The experimental results for the ultra-broadband scenarios are provided in Section 4, followed by a conclusion in Section 5 .

\section{The Bandwidth Requirements on DPD System}

In a DPD system, the bandwidth requirements usually include three parts: the $\mathrm{FB}$, the $\mathrm{Tx}$, and the $\mathrm{BB}$ [12]. In this section, we will provide a detailed analysis for these requirements.

2.1. Conventional DPD System. Generally, in a conventional DPD system, the baseband bandwidth for the DPD modules will require at least five times the input signal bandwidth to fully generate sufficient nonlinear components for satisfactory linearization performance [11], as shown in Figure 2(a). This is because the major part of the predistorted signal will include the components from the fundamental one to the 5th-order one. Here, $500 \mathrm{MHz}$ modulated signal is taken for example. Therefore, at least $2500 \mathrm{MHz}$ bandwidth will be required for the predistorted input signal generation. This will place a huge burden on the baseband bandwidth, since the baseband is usually realized by digital circuits, such as FPGA. 
TABLE 1: The bandwidth requirement for a $500 \mathrm{MHz}$ modulated signal.

\begin{tabular}{lcc}
\hline & Sampling rate (MSPS) & BW $(\mathrm{MHz})$ \\
\hline Tx & (i) Conventional DPD system & $5 \mathrm{x}=2500$ \\
Feedback loop & $5 \mathrm{x}=3200$ & $5 \mathrm{x}=2500$ \\
Baseband & $5 \mathrm{x}=3200$ & $5 \mathrm{x}=2500$ \\
\hline & $5 \mathrm{x}=3200$ & $5 \mathrm{x}=2500$ \\
Tx & (ii) DPD system with reduced feedback loop bandwidth & $<1 \mathrm{x}=500$ \\
Feedback loop & $5 \mathrm{x}=3200$ & $5 \mathrm{x}=2500$ \\
Baseband & $<1 \mathrm{x}=640$ & $1 \mathrm{x}-2 \mathrm{x}=500-1000$ \\
& $5 \mathrm{x}=3200$ & $1 \mathrm{x}-2 \mathrm{x}=500-1000$ \\
Tx & (iii) DPD system with reduced Tx/FB bandwidth & $5 \mathrm{x}=2500$ \\
Feedback loop & $1 \mathrm{x}-2 \mathrm{x}=640-1280$ & $1 \mathrm{x}-2 \mathrm{x}=640-1280$ \\
Baseband & $5 \mathrm{x}=3200$ & \\
\hline
\end{tabular}

Later, a wideband Tx will be involved, in which the digital-to-analog converters (DACs) should be able to support such wide bandwidth. Normally, due to the factor of the filter roll-off, the sampling rate (Fs) for DACs will be slightly higher than the bandwidth value. Here, we set the roll-off factor to 0.28 , and the sampling rate for I/Q baseband signal should be $3200 \mathrm{MSPS}$, accordingly. The rest of RF components should also support such bandwidth to keep the signal transmitted properly.

Similarly, in the $\mathrm{FB}$, due to the spectrum regrowth generated by the PA, enough nonlinear distortion should be captured to correctly describe the output signal. Like the predistorted signal, the output with $5 \mathrm{x}$ the input bandwidth will be considered to be enough for model extraction. Also, the analog-to-digital converters (ADCs) should be able to capture the output with such bandwidth. The details have been listed in Table 1(i). From this table, it can be seen that the $\mathrm{Tx} / \mathrm{FB} / \mathrm{BB}$ will face the huge challenge for $5 \mathrm{G}$ scenarios; that is, the bandwidth requirement is too high.

\subsection{DPD System with Reduced Feedback Loop Bandwidth.} To alleviate the bandwidth pressure, many researchers have started to focus on the bandwidth reduction on the feedback loop [13-21], as shown in Figure 2(b). Guan et al. [13] proposed a bandwidth-constrained least squares-based model extraction method to reduce the feedback loop bandwidth. $\mathrm{Ma}$ et al. [14] proposed a wideband DPD technique using spectral extrapolation of band-limited feedback signal. Liu et al. $[15,16]$ proposed a general DPD architecture using constrained feedback bandwidth for wideband PAs. Tao et al. in [17] proposed a random demodulation based reduced sampling rate method. Wang et al. in $[18,19]$ proposed a novel method using low feedback sampling rate to obtain the DPD coefficients, which is the same as the ones calculated with high sampling rates. Huang et al. in [20] proposed a DPD function synthesis method by using undersampled feedback signal. Wang et al. [21] proposed a low feedback sampling rate digital predistortion technique to linearize a PA excited by a $40 \mathrm{MHz}$ signal with only $2.5 \mathrm{MSPS}$ feedback sampling rate. With the help of these methods, the bandwidth requirement for feedback loop has been significantly reduced.
The successful reduction on FB bandwidth has its own reason. It is because the goal of the feedback loop is only to extract the right DPD coefficients, which provides the possibility of utilizing the undersampled feedback signals. From Figure 2(b), it can be seen that the FB bandwidth can be reduced by different schemes, while the remaining parts of DPD operation will remain unchanged. In this scenario, if the $500 \mathrm{MHz}$ modulated signal is taken again as an example, the FB bandwidth can be decreased to less than the original input signal, but the bandwidth for other parts will still be very wide. The details can be found in Table 1(ii).

2.3. DPD System with Reduced Tx/FB Bandwidth. As mentioned above, even if different methods have been conducted to reduce the bandwidth requirements in the feedback loop, the Tx bandwidth will still be very wide. The reason is that the bandwidth of the predistorted input signal decides the linearization bandwidth. In other words, if the compensation of the distortion within a specified range is required, the predistorted input signal with such range of bandwidth is expected to be provided. From this point of view, the predistorted signal should occupy at least $5 \mathrm{x}$ the input bandwidth to realize the full-band linearization. Therefore, it might be impossible to lower the $\mathrm{Tx}$ bandwidth requirement without changing the DPD structure.

Recently, the band-limited DPD solution [22, 23] has been proposed to resolve the issue of the bandwidth of $\mathrm{Tx} / \mathrm{FB}$, and consequently only $1 \mathrm{x}-2 \mathrm{x}$ input bandwidth will be sufficient for the linearization. It is because this method employs a different DPD architecture by introducing a bandpass filter after PA as shown in Figure 2(c). It is worth mentioning that this filter can be integrated together with the PA design. Based on this structure, the distortion can be divided into two parts: (1) the one within the filter bandwidth and (2) the one out of the filter bandwidth. The former can be removed by the predistorted input signal, while the latter can be eliminated by the filter. Since the distortion bandwidth to be compensated is reduced, the predistorted input signal bandwidth can also be reduced. Therefore, the Tx bandwidth can be narrowed to only support the filter bandwidth. Besides, due to the filter operation, the feedback 


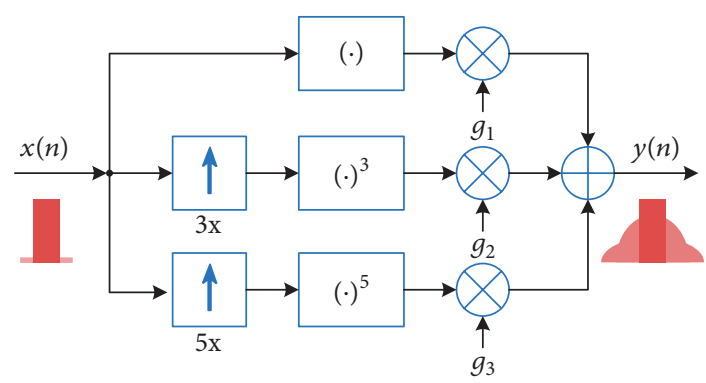

FIGURE 3: The issue of DPD model bandwidth requirements for baseband.

loop bandwidth can also be reduced to the same level. Therefore, this method can realize the bandwidth reduction both on Tx and FB. Here, we take the same example. If the input signal is a $500 \mathrm{MHz}$ signal, the bandwidth requirement for both $\mathrm{Tx}$ and $\mathrm{FB}$ will be only $1 \mathrm{x} \sim 2 \mathrm{x}$ the input bandwidth, that is, $500-1000 \mathrm{MHz}$. The detailed information has been listed in Table 1(iii). From this table, this method still requires high baseband bandwidth for DPD generation and model extraction.

2.4. DPD System with Reduced Baseband Bandwidth. As discussed above, the baseband also needs high bandwidth to correctly generate the predistorted signal without aliasing. It might be affordable for conventional systems but will become a huge burden in ultra-broadband mmWave scenarios.

For better understanding, let us have a look at the structure for DPD model. As shown in Figure 3, to correctly generate the high-order nonlinear components, the baseband bandwidth should be upsampled to multiple times the input bandwidth firstly to avoid aliasing for further operations [610]. Here, we take the Volterra series based model [24] as an example. For 5th-order Volterra kernel, it will occupy $2500 \mathrm{MHz}$ bandwidth, that is, $3200 \mathrm{MSPS}$, if the roll-off factor is taken into consideration. Therefore, proper techniques should be developed to resolve this issue in the baseband as shown in Figure 2(d).

In summary, based on the analysis in this section, the bandwidth requirement can be summarized in Table 1. From this table, with the increase of the bandwidth, the cost for the DPD system gradually becomes unaffordable. In particular, in the promising $5 \mathrm{G}$ mmWave frequency allocation, the wideband modulated signal, for example, $500 \mathrm{MHz}$, will be a basic demand. Therefore, novel DPD architectures should be developed.

\section{Proposed DPD}

In this section, a novel DPD system will be proposed to deal with the $\mathrm{Tx} / \mathrm{FB} / \mathrm{BB}$ bandwidth limitations together.

3.1. Proposed DPD Model Derivation. As described in previous section, the nonlinear model should be employed to correctly describe the PA's behavior and also to form the DPD function. Inside the nonlinear model, there are lots of nonlinear operators, such as 5th-order operator, which will consume huge baseband bandwidth. In particular, for the ultra-broadband mmWave scenario, the situation becomes severer.

To resolve this dilemma, let us rethink it. The decomposed piecewise technique in [25] substitutes high-order operators by several low-order operators. Therefore, if we employ a set of linear piecewise segments to describe the PA nonlinear characteristics and build the DPD functions, the required nonlinearity can be introduced by the piecewise segments and the operations on each segment will be linear. Thus it will not require high baseband bandwidth.

This operation can be expressed as

$$
y(n)=\sum_{i=1}^{N} G_{i} \cdot x_{i}(n),
$$

where $y(n)$ is the predistorted signal, $x_{i}(n)$ is the decomposed input signal, and each segment is weighted by the linear operator $G_{i}$.

To further reduce the bandwidth requirements of Tx and $\mathrm{FB}$, in the proposed method, we are only focusing on the linearization of the in-band distortion and the nearby $\mathrm{OOB}$ distortion. Then, we should embed the filtering operation into the piecewise operation at a low baseband bandwidth.

Therefore, we propose a novel method as shown in Figure 4. Firstly, the original input signal will be processed in parallel:

$$
\begin{aligned}
& x^{(1)}(n)=x(n), \quad n=1,2, \ldots, N s, \\
& x^{(2)}(n)=x(n) * \operatorname{sinc}\left(n+\frac{1}{2}\right), \quad n=1,2, \ldots, N s,
\end{aligned}
$$

where $x^{(1)}(n)$ and $x^{(2)}(n)$ represent two parallel signals and the "sinc" function will be used later in memory effect operation. This is because the description of memory effect in PA will usually require high data rate to achieve better performance. The "sinc" function is one of the best functions to obtain the interpolated point without increasing the data rate, which is only employed in the interim procedures for these two parallel signals.

Then, both of the signals will be decomposed into $N$ segments, and the threshold can be set as $\left[t_{1}, t_{2}, t_{3}, \ldots, t_{N-1}\right]$, depending on the PA's nonlinearity,

$$
\begin{aligned}
& x^{(1)}(n)=\sum_{i=1}^{N} x_{i}^{(1)}(n), \quad n=1,2, \ldots, N s, \\
& x^{(2)}(n)=\sum_{i=1}^{N} x_{i}^{(2)}(n), \quad n=1,2, \ldots, N s,
\end{aligned}
$$

where $x_{i}^{(1)}(n)$ and $x_{i}^{(2)}(n)$ represent the $i$ th segments of the parallel signals, $N$ is the number of piecewise segments, and $N s$ is the number of samples.

Next, the filter operation can be included by separating the finite impulse response (FIR) into two independent 


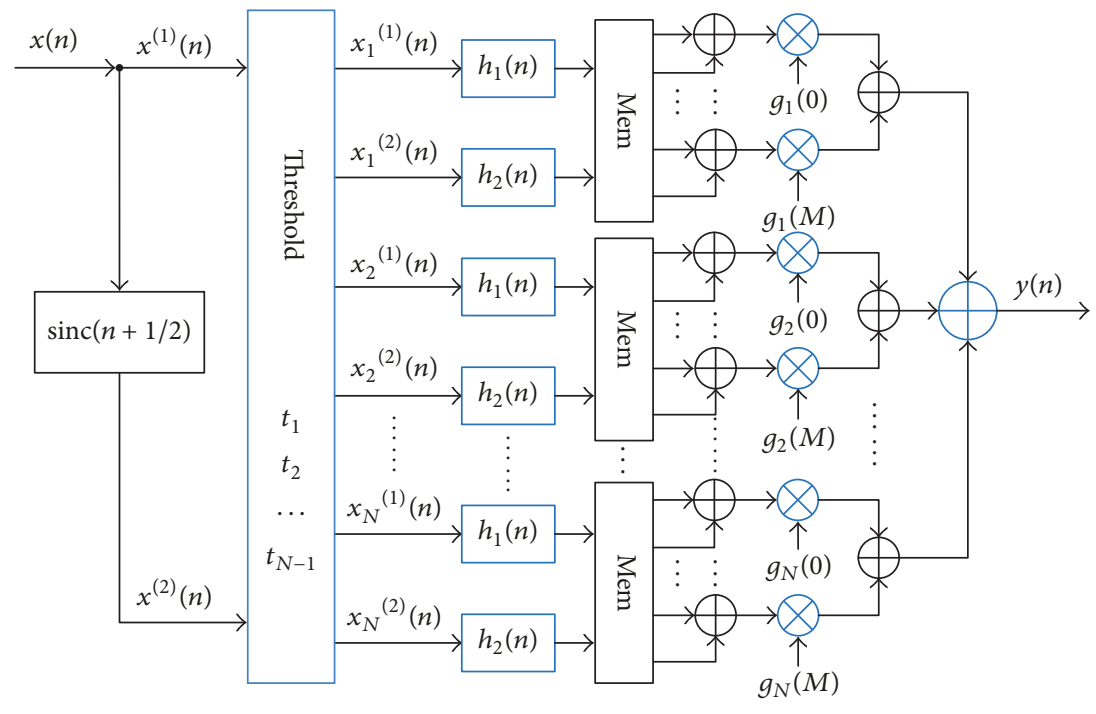

FIGURE 4: The proposed DPD model.

responses to reduce the influence of the nonlinearity caused by the piecewise operation, as expressed as

$$
\begin{array}{ll}
h_{1}(n)=0.4 \operatorname{sinc}[0.4 \cdot(2 k-1)], & k \in\left[-\frac{K-1}{2}, \frac{K-1}{2}\right], \\
h_{2}(n)=0.4 \operatorname{sinc}[0.4 \cdot(2 k)], & k \in\left[-\frac{K-1}{2}, \frac{K-1}{2}\right],
\end{array}
$$

where $h_{1}(n)$ and $h_{2}(n)$ represent two FIR filter responses and $K$ represents the truncated FIR response of the sinc function, and 0.4 is selected due to the roll-off factor. In this way, the filter operations can be embedded:

$$
\begin{aligned}
& u_{i}^{(1)}(n)=x_{i}^{(1)}(n) * h_{1}(n), \\
& u_{i}^{(2)}(n)=x_{i}^{(2)}(n) * h_{2}(n),
\end{aligned}
$$

where $u_{i}^{(1)}(n)$ and $u_{i}^{(2)}(n)$ represent the convolution output of the $i$ th segments of the parallel signals and the designed filters.

Later, since the PA will exhibit the memory effect that is required to be included, we can also process it based on the low bandwidth requirement; that is,

$$
u_{i}(n-m)=u_{i}^{(1)}(n-m)+u_{i}^{(2)}(n-m),
$$

where $u_{i}(n-m)$ represents the $i$ th segments of the $m$ th memory depth combined signal, which is generated in the "Mem" block as shown in Figure 4.

Finally, (1) can be reformulated as

$$
y(n)=\sum_{i=1}^{N} \sum_{m=0}^{M} g_{i}(m) u_{i}(n-m),
$$

where $y(n)$ is the predistorted signal, $g_{i}(m)$ represents the DPD coefficients for the $i$ th segments of the $m$ th memory depth combined signal, $M$ represents the memory depth, and $N$ represents the number of piecewise segments.

The whole DPD model can be illustrated as Figure 4. Since the model structure is still linear-in-parameter, the linear identification methods, for example, least-square, are still valid for the model extraction, as expressed below:

$$
\mathbf{Y}=\mathbf{U C}
$$

where

$$
\begin{aligned}
& \mathbf{U}=\left[\begin{array}{ccc}
u_{1}(1), \ldots, u_{1}(1-M) & \cdots & u_{N}(1), \ldots, u_{N}(1-M) \\
u_{1}(2), \ldots, u_{1}(2-M) & \cdots & u_{N}(2), \ldots, u_{N}(2-M) \\
\vdots & \ddots & \vdots \\
u_{1}(N s), \ldots, u_{1}(N s-M) & \cdots & u_{N}(N s), \ldots, u_{N}(N s-M)
\end{array}\right]
\end{aligned}
$$

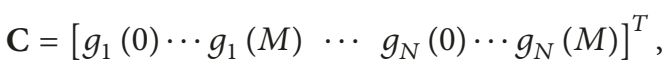

$$
\begin{aligned}
& \mathbf{Y}=\left[\begin{array}{llll}
y(1) & y(2) & \cdots & y(N s)
\end{array}\right]^{T},
\end{aligned}
$$




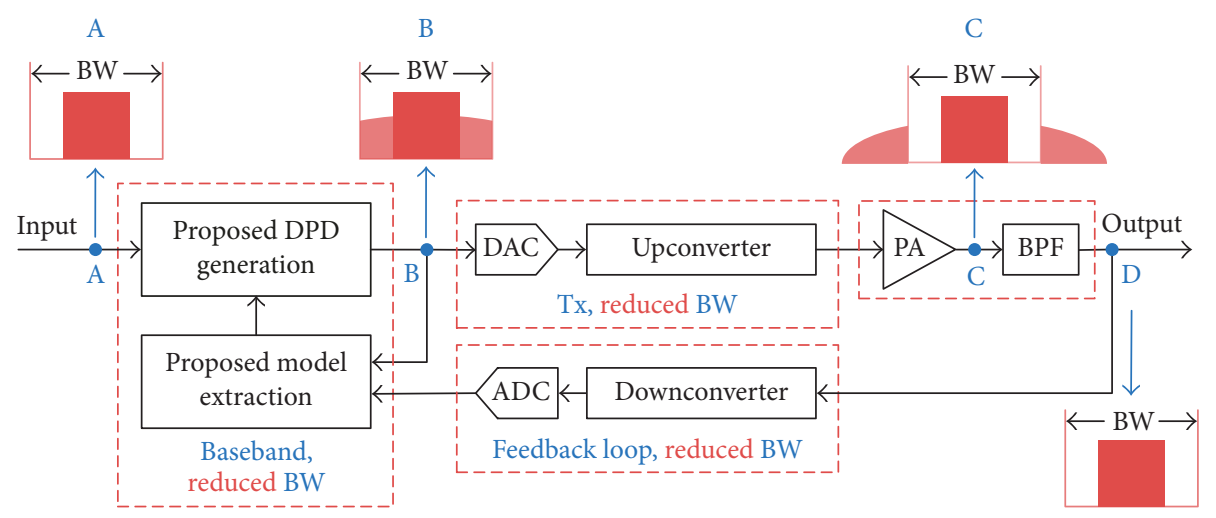

Figure 5: Proposed DPD architecture with reduced FB/Tx/BB bandwidth.

$$
\mathbf{C}=\left(\mathbf{U}^{\mathrm{H}} \mathbf{U}\right)^{-1} \mathbf{U}^{\mathrm{H}} \mathbf{Y}
$$

where $\mathbf{C}$ represents the coefficient matrix, $\mathbf{Y}$ represents the output vector, and $\mathbf{U}$ represents the input matrix. $(\cdot)^{-1}$ denotes the matrix inverse operation and $(\cdot)^{\mathrm{H}}$ is the conjugate transpose.

To correctly obtain the DPD model coefficients, the input and the output of PA will be swapped for the model extraction. In the first iteration, the input matrix $\mathbf{U}$ will be calculated using the PA output signal $y(n)$. For example, in (5), $x(n)$ can be replaced by $y(n)$ to build (9), while the original input $x(n)$ is utilized to construct the output vector $Y$, instead of $y(n)$ in (11). From the second iteration, the input matrix $\mathbf{U}$ will still be calculated by PA output signal $y(n)$, but the output vector $\mathbf{Y}$ will be filled with the predistorted input signal, instead of the original input $x(n)$. Normally, for the DPD operation, it will require several iterations to achieve optimal performance.

From Figure 4, only linear operations are involved in this model and thus baseband bandwidth requirements can be largely reduced, which makes the baseband affordable for ultra-broadband mmWave scenario.

3.2. Complete DPD Architecture. Based on the proposed model, the complete DPD architecture can be constructed as shown in Figure 5. The original input signal (Point A) will be fed into the proposed DPD generation module and then the predistorted input signal (Point $\mathrm{B}$ ) will be generated within limited BB bandwidth, that is, normally, $1 \mathrm{x}-2 \mathrm{x}$ input bandwidth as described above. Since the predistorted signal is limited to a specified bandwidth, the DAC sampling rate can be effectively reduced. And then this signal will be upconverted and fed into the mmWave PA. Since the predistorted input signal only occupies limited bandwidth, the Tx bandwidth can also be largely reduced. Due to this merit, only part of the distortion in PA output will be compensated (Point C). Therefore, a band-pass filter will be employed to suppress the remained out-of-band distortion, which is already at relatively low level. The pass band will occupy $1 \mathrm{x}-2 \mathrm{x}$ input bandwidth and $10-15 \mathrm{~dB}$ distortion suppression should be enough for the stop band. It is worth mentioning that the filter can be codesigned with the PA and integrated into the output matching network of PA. Since the PA output will only occupy limited bandwidth (Point D), only this part will be required to be fed into the feedback loop, which will lead to a significant reduction on the bandwidth of the feedback loop. Both the input and output signal will be sent into the model extraction block. In this module, all the processing bandwidth is also limited into a specified range.

In summary, in the proposed DPD architecture, all the bandwidth requirements including the Tx, feedback loop, and the baseband will be significantly reduced. The detailed performance can be found in Table 2 .

3.3. Complexity Analysis and Discussion. Since there are only linear operations in the model, the computational cost will be pretty low. Usually, the model complexity can be evaluated in terms of the number of the coefficients. Therefore, for the proposed model, the coefficient number mainly depends on the number of the piecewise segments and the memory depth; that is,

$$
\text { Coeff. Num }=N *(M+1),
$$

where $N$ represents the number of piecewise segments and $M$ represents the memory depth. Take $N=10$ and $M=5$, for example; there are only 60 coefficients in total.

In summary, compared to the conventional model, the proposed DPD architecture can provide the following advantages:

(1) Low bandwidth requirements are required for the whole DPD system, including Tx, FB, and BB, which make the ultra-broadband mmWave DPD affordable.

(2) Low complexity is required for model construction with linear operations only, which makes the implementation in FPGA simple.

(3) Linear operation property will keep the computation for model extraction stable. 
TABLE 2: The bandwidth requirement in proposed DPD system for a $500 \mathrm{MHz}$ modulated signal.

\begin{tabular}{lcc}
\hline & Sampling rate (MSPS) & BW (MHz) \\
\hline Tx & $1 \mathrm{x}-2 \mathrm{x}=640-1280$ & $1 \mathrm{x}-2 \mathrm{x}=500-1000$ \\
Feedback loop & $1 \mathrm{x}-2 \mathrm{x}=640-1280$ & $1 \mathrm{x}-2 \mathrm{x}=500-1000$ \\
Baseband & $1 \mathrm{x}-2 \mathrm{x}=640-1280$ & $1 \mathrm{x}-2 \mathrm{x}=500-1000$ \\
\hline
\end{tabular}

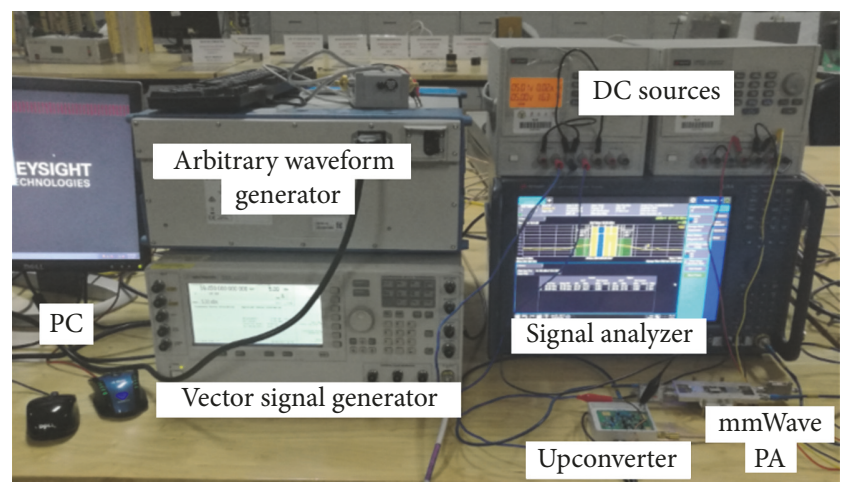

FIGURE 6: The test bench.

\section{Experimental Results}

In this section, the test bench will be set up to validate the proposed idea in ultra-broadband mmWave scenarios.

4.1. Test Bench Setup. The test bench was set up as shown in Figure 6. A wideband modulated signal was generated by the software Matlab in PC, downloaded into the arbitrary waveform generator (Keysight M8190). Then, it will be upconverted by an in-house designed upconverter module and finally fed into an in-housed designed mmWave PA module@41GHz. The upconverter module in Figure 7 covers a frequency range from 40.5 to $43.5 \mathrm{GHz}$ and supports bandwidth up to $800 \mathrm{MHz}$. And the PA module as shown in Figure 8 is fabricated by GaAs PHEMT MMIC (Hittite HMC 1016) with P1dB of $24 \mathrm{dBm}$. Then, the output signal is captured by the spectrum analyzer (Keysight N9040B) with the specified observation bandwidth.

4.2. Test Case 1: $80 \mathrm{MHz}$ 4-Carrier LTE Signal. In this scenario, a 4-carrier $80 \mathrm{MHz}$ LTE signal with the peak-toaverage power ratio (PAPR) of $7.5 \mathrm{~dB}$ is employed as the test signal. The proposed model is set with the threshold

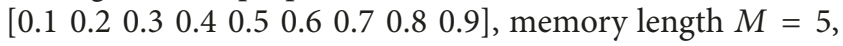
with only 60 coefficients. The average output power is set at $15 \mathrm{dBm}$ and the observation bandwidth is set as $144 \mathrm{MHz}$ for proposed method and $576 \mathrm{MHz}$ for conventional method for comparison.

Figures 9(a) and 9(b) show the measured AM/AM and $\mathrm{AM} / \mathrm{PM}$ characteristics. It can be seen that the nonlinear distortion can be effectively removed by the proposed method.

Figure 9(c) shows the performance comparison for $80 \mathrm{MHz}$ modulated signal between the proposed and conventional techniques. The proposed method with only $144 \mathrm{MHz}$ bandwidth can achieve the similar performance within the

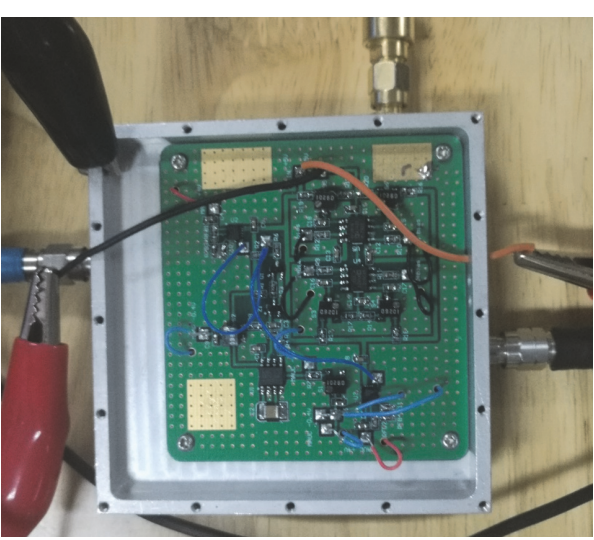

Figure 7: The upconverter module.

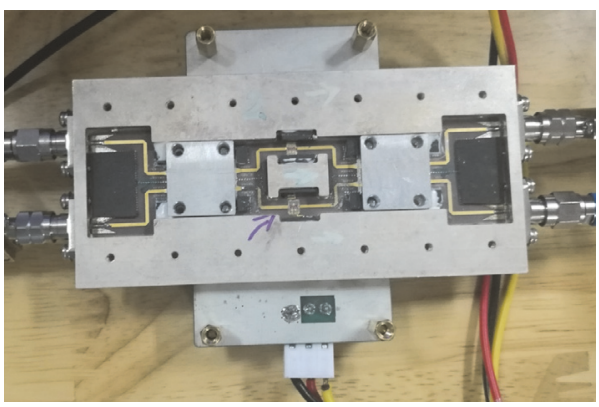

FIGURE 8: The mmWave power amplifier.

interested bandwidth, compared to the conventional method with $576 \mathrm{MHz}$ bandwidth.

The performance in detail has been listed in Table 3 . The adjacent channel power ratio (ACPR) for the proposed method can be improved by $15 \mathrm{~dB} / 16 \mathrm{~dB}$, compared to the one without DPD. The normalized mean square error (NMSE) can be also optimized by $16 \mathrm{~dB}$, which gives a good indication for the performance in time domain.

4.3. Test Case 2: $320 \mathrm{MHz}$ 4-Carrier CA Signal. In this scenario, a $320 \mathrm{MHz} 4$-carrier LTE signal with the $7.5 \mathrm{~dB}$ PAPR is used as the test signal to evaluate the performance for forthcoming ultra-broadband mmWave scenario in 5G applications. The proposed model is also set with the threshold

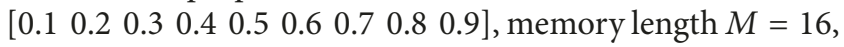
with 170 coefficients. The average output power is set at $15 \mathrm{dBm}$ and the observation bandwidth is set as $576 \mathrm{MHz}$ for proposed method. However, due to the bandwidth limitation of the test bench, the conventional method will require very large $\mathrm{Tx} / \mathrm{FB} / \mathrm{BB}$ bandwidth and thus was not carried out in $320 \mathrm{MHz}$ signal test.

Figures 10(a) and 10(b) give an illustration for measured $\mathrm{AM} / \mathrm{AM}$ and $\mathrm{AM} / \mathrm{PM}$ characteristics. It can be seen that the nonlinear distortion can be successfully compensated, but there is still some memory effect left. This is because the noise floor for ultra-broadband mmWave test bench is pretty high, which will inevitably affect the DPD performance. Further investigations will be carried out in future work to optimize the noise floor performance. 


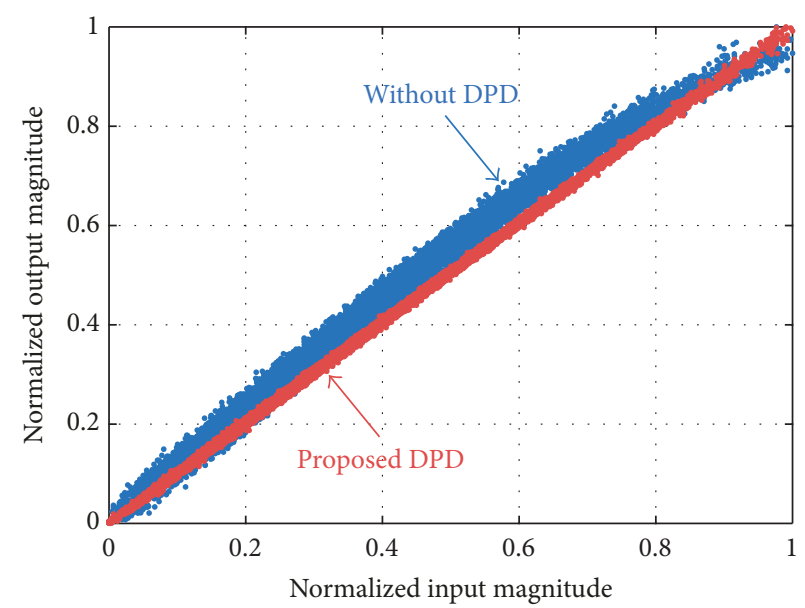

(a)

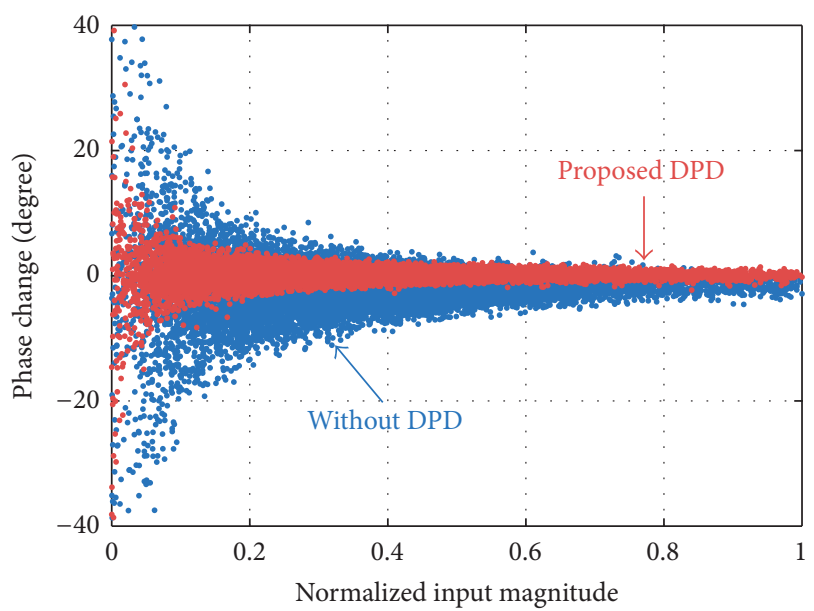

(b)

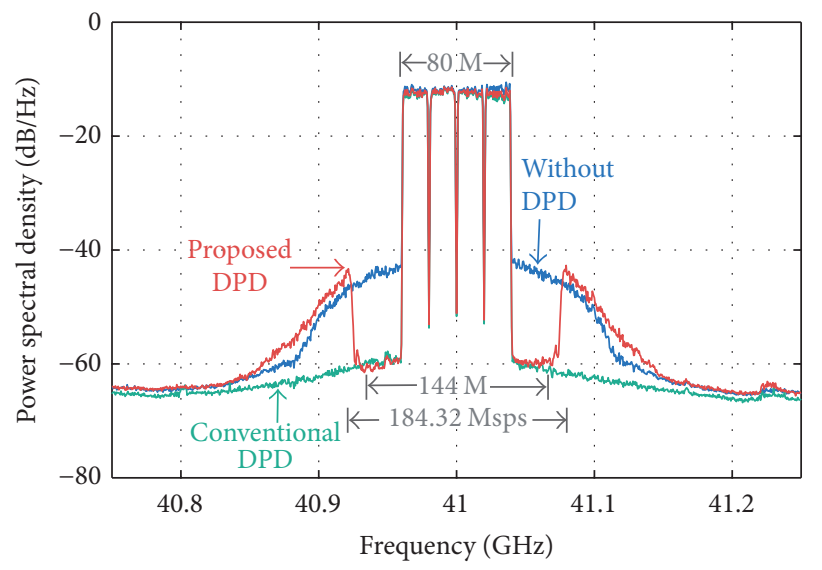

(c)

FIGURE 9: Measured performance for $80 \mathrm{MHz}$ modulated signal. (a) AM/AM. (b) AM/PM. (c) Power spectral density.

TABLE 3: Measured performance for $80 \mathrm{MHz}$ signal.

\begin{tabular}{lccccc}
\hline & ACPR $(\mathrm{dBc})$ & NMSE $(\mathrm{dB})$ & NRMSE $(\%)$ & Sampling rate (MSPS) & Analysis BW (MHz) \\
\hline No DPD & $-32.25 /-31.63$ & -18.47 & 6.8 & NA & NA \\
Conv. DPD & $-46.96 /-47.90$ & -33.15 & 1.88 & 737.28 & 576 \\
Proposed DPD & $-47.64 /-47.47$ & -34.63 & 1.66 & 184.32 & 144 \\
\hline
\end{tabular}

Figure 10(c) shows the measured power spectrum density with/without DPD. The details can be found in Table 4 . The proposed method can achieve around 10/12 dB improvements for ACPR value and $12 \mathrm{~dB}$ improvements for NMSE performance. It is noticeable that, by employing the proposed DPD system, the $320 \mathrm{MHz}$ signal @ $41 \mathrm{GHz}$ can be effectively linearized by only using $576 \mathrm{MHz}$ bandwidth. This merit can reduce not only the requirement for $\mathrm{Tx} / \mathrm{FB}$, but also the $\mathrm{BB}$ requirement, which will effectively overcome the main bottleneck in the mmWave DPD system.

4.4. Model Comparison. In this part, the proposed method will be compared with the band-limited DPD technique (BLDPD) [22] for both $80-\mathrm{MHz}$ and $320-\mathrm{MHz}$ scenarios.

Figure 11 shows the measured performance for both scenarios. From Figure 11, it can be seen that both BL-DPD and proposed DPD can achieve similar performance, which provides a validation for the proposed method. However, the bandwidth requirements are different. Table 5 illustrates the bandwidth comparisons for both methods in detail. The requirements for $\mathrm{ADC} / \mathrm{DAC}$ sampling rates and $\mathrm{Tx} / \mathrm{FB}$ bandwidth are all the same. However, compared to the BL-DPD, the proposed method can further reduce the baseband rate to keep it at the same level as other bandwidth requirements, which proves that the proposed method can provide the comprehensive bandwidth reduction for the Tx, feedback loop, and baseband.

\section{Conclusion}

In this paper, a novel DPD technique was proposed to significantly reduce the bandwidth requirements for the 


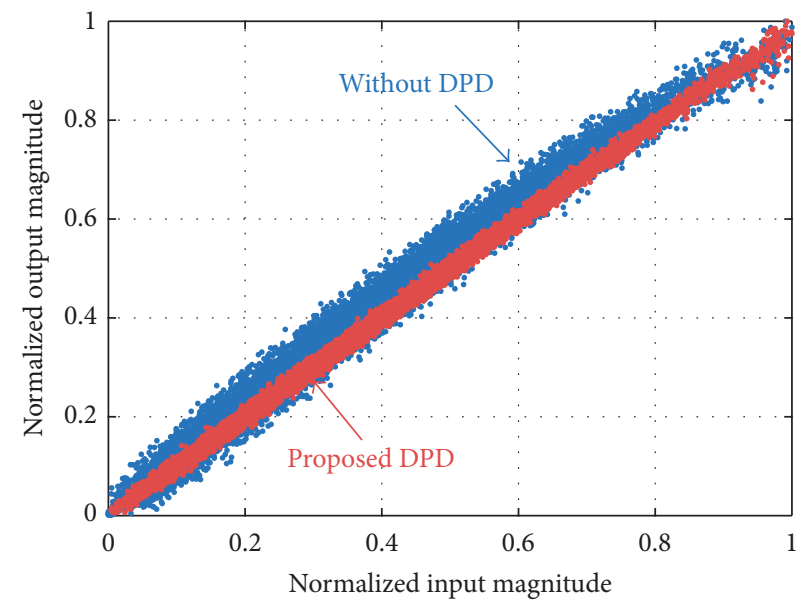

(a)

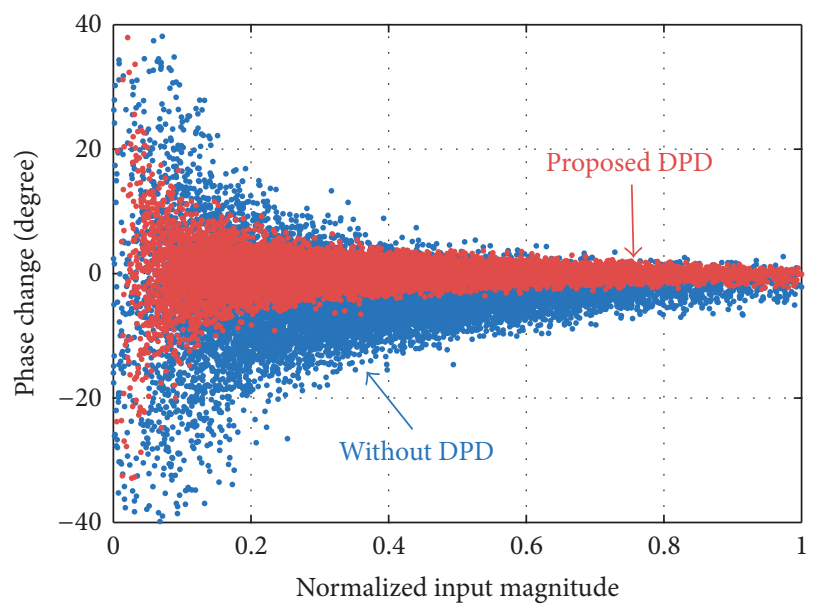

(b)

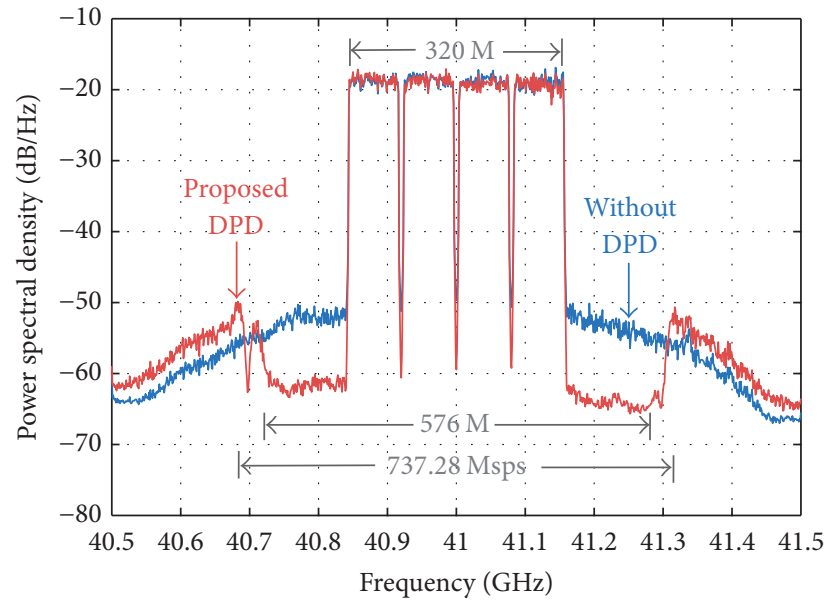

(c)

Figure 10: Measured performance for $320 \mathrm{MHz}$ modulated signal. (a) AM/AM. (b) AM/PM. (c) Power spectral density.

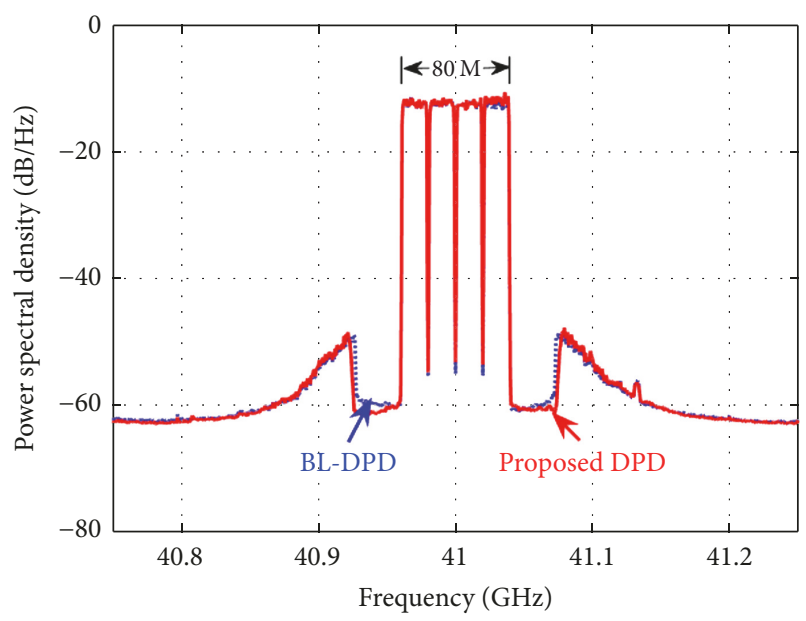

(a)

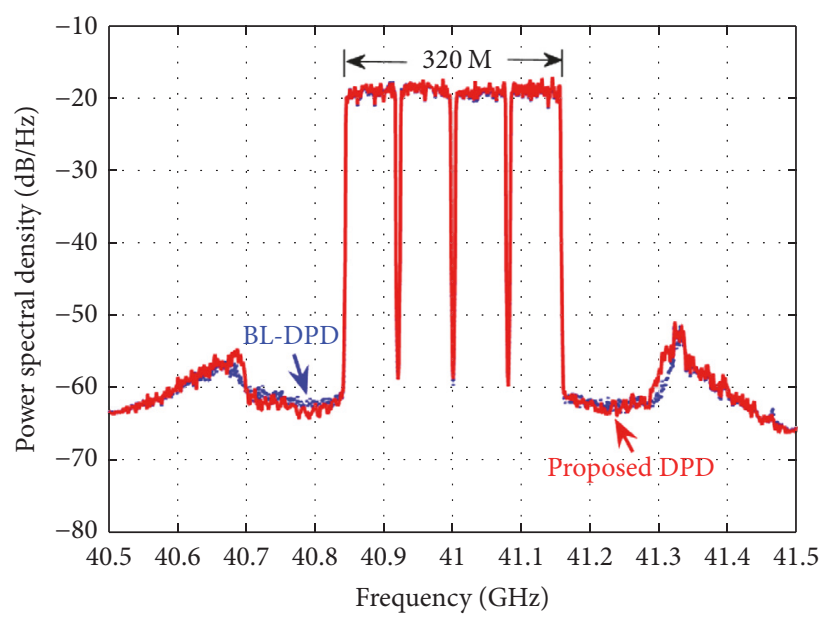

(b)

FIGURE 11: Measured performance comparison for (a) $80 \mathrm{MHz}$ modulated signal and (b) $320 \mathrm{MHz}$ modulated signal. 
TABLE 4: Measured performance for $320 \mathrm{MHz}$ signal.

\begin{tabular}{lccccc}
\hline & ACPR $(\mathrm{dBc})$ & NMSE $(\mathrm{dB})$ & NRMSE $(\%)$ & Sampling rate (MSPS) & Analysis BW $(\mathrm{MHz})$ \\
\hline No DPD & $-33.37 /-33.88$ & -18.37 & 10.22 & NA & NA \\
Proposed DPD & $-42.92 /-45.04$ & -30.13 & 3.1 & 737.28 & 576 \\
\hline
\end{tabular}

TABLE 5: Bandwidth comparison for BL-DPD and proposed DPD.

\begin{tabular}{|c|c|c|c|c|c|}
\hline & Baseband rate (MSPS) & ADC sampling rate (MSPS) & Tx BW (MHz) & DAC sampling rate (MSPS) & FB BW $(\mathrm{MHz})$ \\
\hline \multicolumn{6}{|c|}{$80 \mathrm{MHz}$ scenario } \\
\hline BL-DPD & 737.28 & 184.32 & 144 & 184.32 & 144 \\
\hline Proposed DPD & 184.32 & 184.32 & 144 & 184.32 & 144 \\
\hline \multicolumn{6}{|c|}{$320 \mathrm{MHz}$ scenario } \\
\hline BL-DPD & 2949.12 & 737.28 & 576 & 737.28 & 576 \\
\hline Proposed DPD & 737.28 & 737.28 & 576 & 737.28 & 576 \\
\hline
\end{tabular}

Tx, feedback loop, and baseband in the context of ultrabroadband mmWave scenarios. This proposed technique will provide the capability of linearizing an ultra-broadband mmWave PA with affordable resources, which can largely extend the DPD regime into $5 \mathrm{G}$ mmWave era.

\section{Conflicts of Interest}

The authors declare that there are no conflicts of interest regarding the publication of this paper.

\section{Acknowledgments}

The authors would like to thank Keysight Technologies for providing the software and hardware support. This work was supported in part by the National Natural Science Foundation of China (NSFC) under Grant 61601117 and the Natural Science Foundation of Jiangsu Province under Grant BK20160698.

\section{References}

[1] E. Dahlman, G. Mildh, S. Parkvall et al., "5G wireless access: Requirements and realization," IEEE Communications Magazine, vol. 52, no. 12, pp. 42-47, 2014.

[2] M. J. Marcus, "5G and 'iMT for 2020 and beyond," IEEE Wireless Communications Magazine, vol. 22, no. 4, 2015.

[3] J. Wood, Behavioral Modeling and Linearization of RF Power Amplifiers, Artech House, Norwood, MA, USA, 2014.

[4] F.-L. Guo, Digital Front End, Cambridge Univ. Press, UK, 2011.

[5] L. Guan and A. Zhu, "Green communications," IEEE Microw. Mag, vol. 15, no. 7, pp. 84-99, 2014.

[6] A. Zhu, J. C. Pedro, and T. J. Brazil, "Dynamic deviation reduction-based Volterra behavioral modeling of RF power amplifiers," IEEE Transactions on Microwave Theory and Techniques, vol. 54, no. 12, pp. 4323-4332, 2006.

[7] W. Cao and A. Zhu, "A Modified Decomposed Vector RotationBased Behavioral Model with Efficient Hardware Implementation for Digital Predistortion of RF Power Amplifiers," IEEE Transactions on Microwave Theory and Techniques, vol. 65, no. 7, pp. 2443-2452, 2017.
[8] A. Zhu, "Decomposed vector rotation-based behavioral modeling for digital predistortion of RF power amplifiers," IEEE Transactions on Microwave Theory and Techniques, vol. 63, no. 2, pp. 737-744, 2015.

[9] J. Kim and K. Konstantinou, "Digital predistortion of wideband signals based on power amplifier model with memory," IEEE Electronics Letters, vol. 37, no. 23, pp. 1417-1418, 2001.

[10] D. R. Morgan, Z. Ma, J. Kim, M. G. Zierdt, and J. Pastalan, "A generalized memory polynomial model for digital predistortion of RF power amplifiers," IEEE Transactions on Signal Processing, vol. 54, no. 10, pp. 3852-3860, 2006.

[11] F. M. Ghannouchi and O. Hammi, "Behavioral modeling and predistortion," IEEE Microwave Magazine, vol. 10, no. 7, pp. 5264, 2009.

[12] J. Wood, "System-level design considerations for digital predistortion of wireless base station transmitters," IEEE Transactions on Microwave Theory and Techniques, vol. 65, no. 5, pp. 1880-1890, 2017.

[13] L. Guan, C. Yu, and A. Zhu, "Bandwidth-constrained least squares-based model extraction for band-limited digital predistortion of RF power amplifiers," in Proceedings of the International Workshop on Integrated Nonlinear Microwave and Millimetre-Wave Circuits, INMMIC '12, Ireland, September 2012.

[14] Y. Ma, Y. Yamao, Y. Akaiwa, and K. Ishibashi, "Wideband digital predistortion using spectral extrapolation of band-limited feedback signal," IEEE Transactions on Circuits and Systems I: Regular Papers, vol. 61, no. 7, pp. 2088-2097, 2014.

[15] Y. Liu, W. Pan, S. Shao, and Y. Tang, "New digital predistortion for wideband power amplifiers with constrained feedback bandwidth," IEEE Microwave and Wireless Components Letters, vol. 23, no. 12, pp. 683-685, 2013.

[16] Y. Liu, W. Pan, S. Shao, and Y. Tang, "A general digital predistortion architecture using constrained feedback bandwidth for wideband power amplifiers," IEEE Transactions on Microwave Theory and Techniques, vol. 63, no. 5, pp. 1544-1555, 2015.

[17] W. Tao, H. Wang, C. Zhou, G. Li, and F. Liu, "A random demodulation based reduced sampling rate method for wideband digital predistortion," in Proceedings of the Asia-Pacific Microwave Conference, APMC '15, China, December 2015.

[18] Z. Wang, S. Ibrahim, H. Su, and R. Farrell, "Generalised digital predistortion of RF power amplifiers with low-rate feedback signal," in Proceedings of the 46th European Microwave Conference, EuMC '16, pp. 831-834, UK, October 2016. 
[19] Z. Wang, L. Guan, and R. Farrell, "Undersampling ObservationBased Compact Digital Predistortion for Single-Chain Multiband and Wideband Direct-to-RF Transmitter," IEEE Transactions on Microwave Theory and Techniques, vol. 65, no. 12, pp. 5274-5283, 2017.

[20] H. Huang, P. Mitran, and S. Boumaiza, "Digital Predistortion Function Synthesis using Undersampled Feedback Signal," IEEE Microwave and Wireless Components Letters, vol. 26, no. 10, pp. 855-857, 2016.

[21] Z. Wang, W. Chen, G. Su, F. M. Ghannouchi, Z. Feng, and Y. Liu, "Low Feedback Sampling Rate Digital Predistortion for Wideband Wireless Transmitters," IEEE Transactions on Microwave Theory and Techniques, vol. 64, no. 11, pp. 3528-3539, 2016.

[22] C. Yu, L. Guan, E. Zhu, and A. Zhu, "Band-limited volterra series-based digital predistortion for wideband RF power amplifiers," IEEE Transactions on Microwave Theory and Techniques, vol. 60, no. 12, pp. 4198-4208, 2012.

[23] C. Yu, L. Guan, and A. Zhu, "Band-limited Volterra series-based behavioral modeling of RF power amplifiers," in Proceedings of the IEEE MTT-S International Microwave Symposium, IMS '12, pp. 1-3, Canada, June 2012.

[24] M. Schetzen, The Volterra and Wiener Theories of Nonlinear Systems, Krieger, Melbourne, FL, USA, 2006.

[25] A. Zhu, P. J. Draxler, C. Hsia, T. J. Brazil, D. F. Kimball, and P. M. Asbeck, "Digital predistortion for envelope-tracking power amplifiers using decomposed piecewise volterra series," IEEE Transactions on Microwave Theory and Techniques, vol. 56, no. 10, pp. 2237-2247, 2008. 


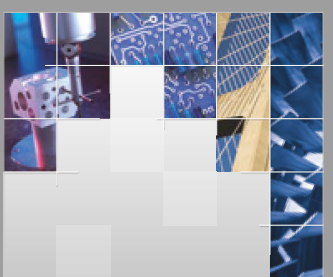

\section{Enfincering}
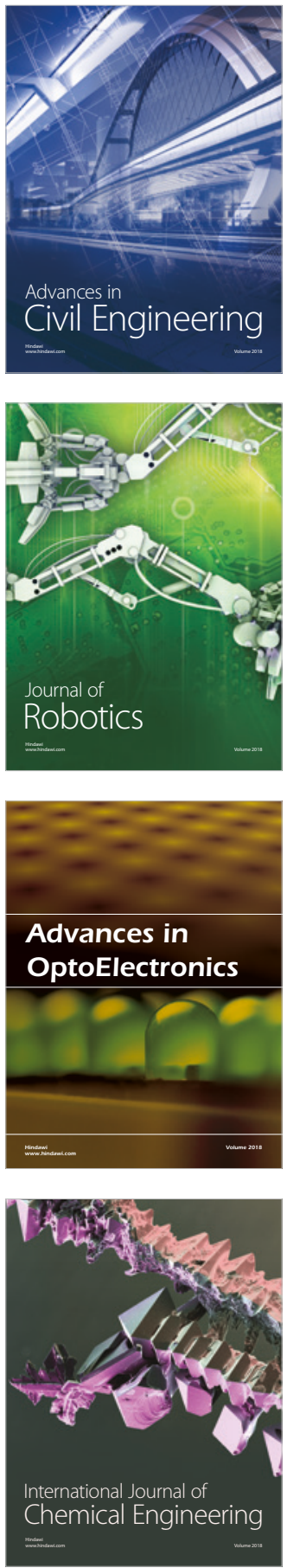

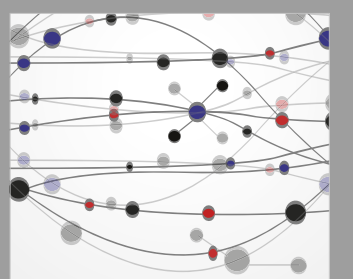

\section{Rotating \\ Machinery}

The Scientific World Journal

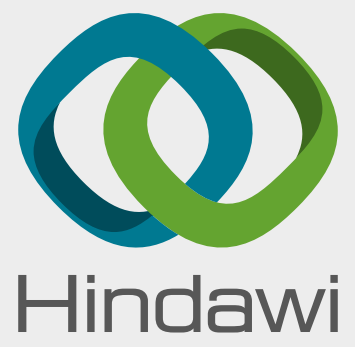

Submit your manuscripts at

www.hindawi.com
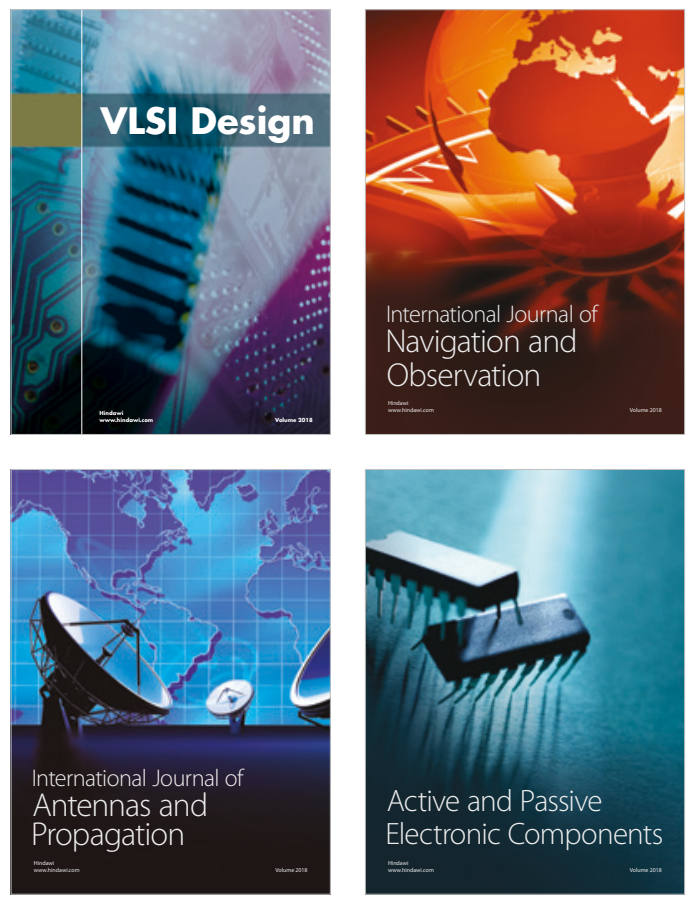
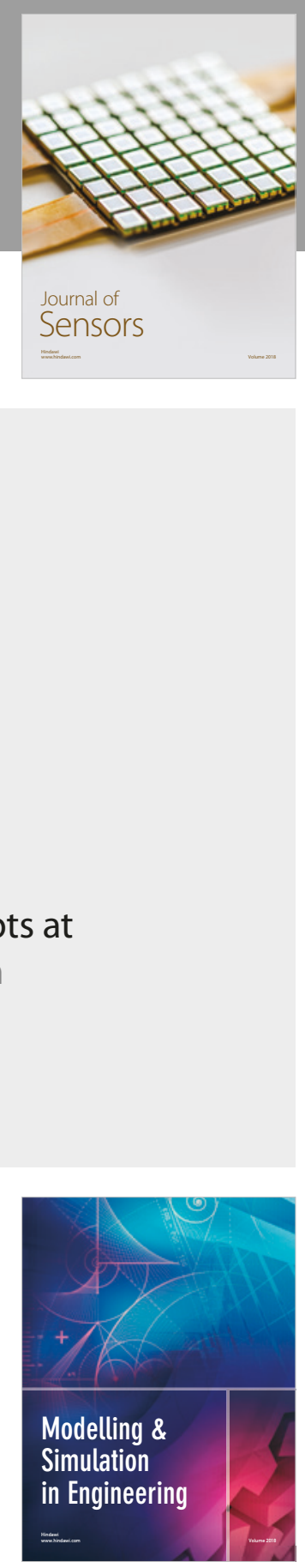

\section{Advances \\ Multimedia}
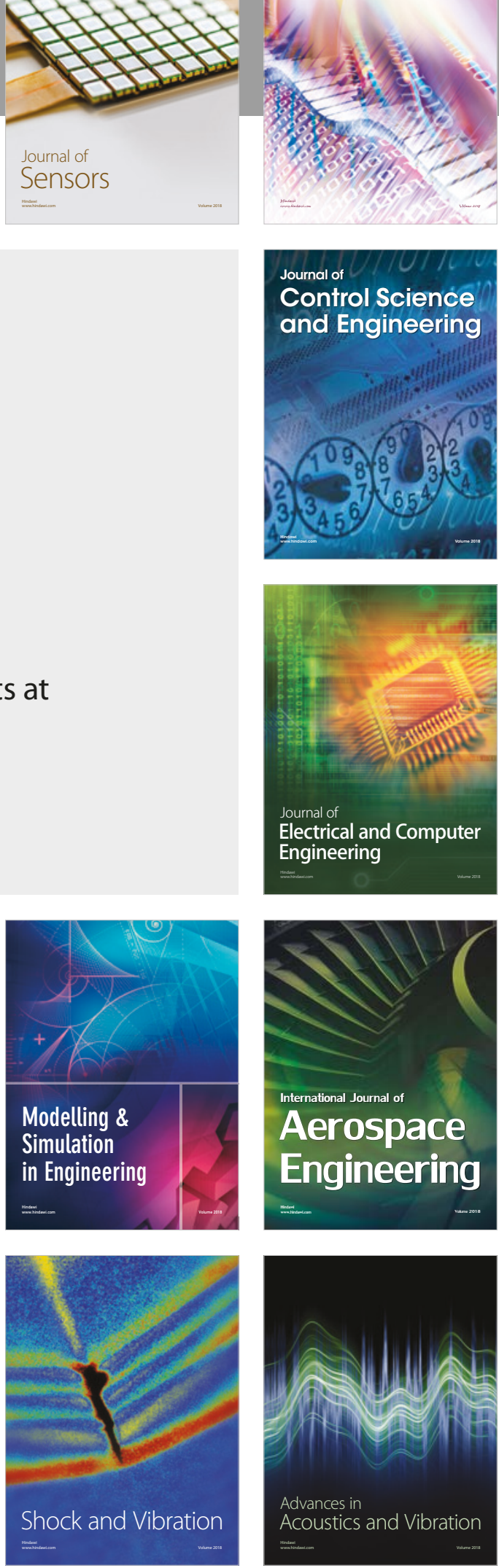